Publ. RIMS, Kyoto Univ.

13 (1977), 159-165

\title{
Notes on Minimality and Ergodicity of Compact Abelian Group Extensions of Dynamics
}

\author{
By \\ Motosige OsIKaWA*
}

\section{$\S$ 0. Introduction and Definitions}

W. Parry [5] introduced the notion of a $G$-extension of a topological dynamics, where $G$ is a compact abelian group, and gave necessary and sufficient conditions for a $G$-extension of a minimal (respectively uniquely ergodic) topological dynamics to be minimal (uniquely ergodic). In the first part of this paper a proof of the Minimality Theorem of W. Parry without his "simple free" condition is given. In the purely measuretheoretic case W. Parry [6] introduced the notion of $G$-extension of type $\sigma$, where $\sigma$ is an automorphism of $G$, and spectrally analysed it. In the second part of this paper a necessary and sufficient condition for a $G$ extension of an ergodic measure-preserving dynamics to be ergodic is shown. As particular cases of this result we have well-known necessary and sufficient conditions for a translation, a group-automorphism and an affine transformation on a compact group to be ergodic.

Throughout, $G$ and $\widehat{G}$ will respectively denote a compact abelian metric group and its character-group. An element $\gamma$ of $\widehat{G}$ is called $n$ periodic with respect to an automorphism $\sigma$ of $G$ if $r \sigma \neq \gamma, \cdots, \gamma \sigma^{n-1} \neq \gamma$ and $r \sigma^{n}=\gamma(n \geqq 1)$. A topological dynamics $(X, S)$ is a compact metric space $X$, together with a homeomorphism $S$. A topological dynamics $\left(X_{1}, S_{1}\right)$ is conjugate to $(X, S)$ if there is a homeomorphism $\tau$ of $X$ onto $X_{1}$ such that the diagram

Cummunicated by H. Araki, September 3, 1976.

* Department of Mathematics, College of General Education, Kyushu University, Fukuoka 810 , Japan. 


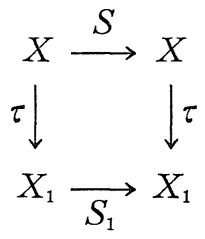

commutes. A set $F$ is $S$-invariant if $S F=F$. An $S$-invariant closed set $F$ is $S$-minimal if the only $S$-invariant closed subsets of $F$ are $F$ and $\Phi$. $(X, S)$ is minimal if $X$ is $S$-minimal. Denote respectively by $C(X)$ and $C(X, K)$, the set of all continuous complex-valued functions defined on $X$ and the set of all functions in $C(X)$ with absolute value 1 .

A continuous $G$-action on $X$ is a continuous map $\chi$ of $G \times X$ onto $X$ such that $\chi(g, \chi(h, x))=\chi(g h, x)$ for $x$ in $X$ and $g, h$ in $G$ and $\chi(e, x)$ $=x$ for $x$ in $X$ where $e$ is the identity element of $G$. If the map $\chi$ is understood we shall write $g x$ for $\chi(g, x)$. If $(X, S)$ is a topological dynamics such that $S$ commutes with the $G$-action (i.e. $S g x=g S x$ for $x$ in $X$ and $g$ in $G$ ) then $S$ induces the homeomorphism $S^{\prime}$ on the $G$-orbit space $X / G$ defined by $S^{\prime} G(x)=G(S x)$ where $G(x)=\{g x ; g \in G\}$. If a topological dynamics $\left(X_{1}, S_{1}\right)$ is conjugate to the topological dynamics $\left(X / G, S^{\prime}\right)$ we shall say that $(X, S)$ is a $G$-extension of $\left(X_{1}, S_{1}\right)$. (W. Parry [5]).

A measure-preserving dynamics $(\Omega, \mu, \varphi)$ (in this paper) is a Lebesgue measure space $(\Omega, \mu), \mu(\Omega)=1$, together with a bimeasurable bijection $\varphi$ such that $\mu(\varphi \Lambda)=\mu(\Lambda)$ for any measurable set $\Lambda$. For simplicity of notation, expressions involving sets or functions will be stated disregarding sets of measure zero. A measure-preserving dynamics $\left(\Omega_{1}, \mu_{1}, \varphi_{1}\right)$ is conjugate to $(\Omega, \mu, \varphi)$ if there is a bimeasurable, measure-preserving bijection $\tau$ from $(\Omega, \mu)$ onto $\left(\Omega_{1}, \mu_{1}\right)$ such that the diagram

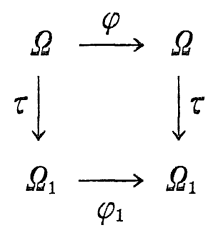

commutes. $(\Omega, \mu, \varphi)$ is ergodic if every measurable function $f$ with $f(\varphi \omega)$ $=f(\omega)$ is constant. Denote by $L^{2}(\Omega, \mu)$ the set of all square-integrable functions on $\Omega$. A measurable $G$-action on $(\Omega, \mu)$ is a measurable map 
$\chi$ of $G \times \Omega$ onto $\Omega$ such that $\chi(g, \chi(h, \omega))=\chi(g h, \omega)$ for $\omega$ in $\Omega$ and $g, h$ in $G, \chi(e, x)=x$ for $x$ in $\Omega$, and $\mu(\chi(g, \Lambda))=\mu(\Lambda)$ for any measurable set $\Lambda$ and any $g$ in $G$. If the map $\chi$ is understood we shall write $g \omega$ for $\chi(g, \omega)$. If $(\Omega, \mu, \varphi)$ is a measure-preserving dynamics such that $\varphi g \omega=\sigma(g) \varphi \omega$ for $\omega$ in $\Omega$ and $g$ in $G$ for some automorphism $\sigma$ of $G$, then $\varphi$ induces the measure-preserving transformation $\varphi^{\prime}$ on the $G$ orbit space $\Omega / G$. If a measure-preserving dynamics $\left(\Omega_{1}, \mu_{1}, \varphi_{1}\right)$ is conjugate to the $\left(\Omega / G, \mu_{\Omega / G}, \varphi^{\prime}\right)$ we shall say that $(\Omega, \mu, \varphi)$ is a $G$-extension of type $\sigma$ of $\left(\Omega_{1}, / /_{1}, \varphi_{1}\right)$. (W. Parry [6]).

\section{§1. Minimality of a $G$-extension}

Lemma 1. Let $(X, S)$ be a G-extension of a minimal topological dynamics. Then for any S-minimal set $C, g C$ is $S$-minimal for any $g$ in $G$ and $X=\bigcup_{g \in G} g C$.

Proof. It is easy to see that $g C$ is $S$-minimal for any $g$ in $G$. We denote by $\pi$ the map from $(X, S)$ to the minimal topological dynamics $\left(X_{1}, S_{1}\right)$ defined by $\pi x=\tau^{-1} G(x)$. The set $\underset{g \in G}{\cup} g C$ is closed and $S$-invariant and the set $\pi\left(\cup_{g \in G} g C\right)$ is closed and $S_{1}$-invariant. From the minimality of $\left(X_{1}, S_{1}\right)$ we have $\pi\left(\cup_{g \in G} g C\right)=X_{1}$. Therefore we have $\underset{g \in G}{\cup} g C=X$.

q.e.d.

Lemma 2. Let $Y$ be a compact topological space on which there is a continuous $G$-action such that $Y=\{g y ; g \in G\}$ for some (any) point $y$ in $Y$. And let $\Gamma$ be the set of all $\gamma$ in $\widehat{G}$ such that there exists an $f_{\tau}$ in $C(Y, K)$ with $f_{r}(g y)=\gamma(g) f_{r}(y)$ for $y$ in $Y$ and $g$ in $G$. Then Sor $h$ in $G, \gamma(h)=1$ for any $\gamma$ in $\Gamma$ if and only if $h y=y$ for any $y$ in $Y$. In particular, $\Gamma=\{1\}$ if and only if $Y$ is one point space.

Proof. For $f$ in $C(Y)$ and $\gamma$ in $\widehat{G}$, put $f_{r}(y)=\int \gamma(g) f\left(g^{-1} y\right) d g$ where $d g$ is the Haar measure on $G$, then $f_{r}(g y)=\gamma(g) f_{r}(y)$ and $f_{r}=0$ for $r$ not in $\Gamma$. Now $\gamma(h)=1$ for any $\gamma$ in $\Gamma$, iff $f_{\gamma}(h y)=f_{\gamma}(y)$ for $y$ in $Y$, any $f$ in $C(Y)$ and any $\gamma$ in $\widehat{G}$, iff $\int \gamma(g)\left\{f\left(g^{-1} h y\right)-f\left(g^{-1} y\right)\right\} d g=0$ 
for $y$ in $Y$, any $f$ in $C(Y)$ and any $\gamma$ in $\widehat{G}$, iff $f(h y)=f(y)$ for $y$ in $Y$, any $f$ in $C(Y)$. All these hold iff $h y=y$ for $y$ in $Y$. q.e.d.

Theorem 1. Let $(X, S)$ be a G-extension of a minimal topological dynamics. Then $(X, S)$ is not minimal if and only if there exists $a \gamma$ in $\widehat{G}, \gamma \neq 1$ and $f$ in $C(X, K)$ such that $f(g x)=\gamma(g) f(x)$ and $f(S x)=f(x)$ for any $x$ in $X$ and any $g$ in $G$.

Proof. Note that the quotient space $X / C$ is Hausdorff (and compact) and apply Lemma 1 and Lemma 2.

q.e.d.

Corollary 1. (H. Fürstenberg [2], W. Parry [5]). Let $(X, S)$ be a minimal topological dynamics and $\alpha$ be a continuous G-valued function defined on $X . \quad \widetilde{S}$ is a homeomorphism of the product space $X \times G$ defined by

$$
\widetilde{S}(x, g)=(S x, \alpha(x) g),(x, g) \text { in } X \times G .
$$

Then the topological dynamics $(X \times G, \widetilde{S})$ is not minimal if and only if there exists $a r$ in $\widehat{G}, r \neq 1$ and an $f$ in $C(X, K)$ such that

$$
\gamma(\alpha(x)) f(S x)=f(x) \text { for all } x \text { in } X .
$$

Proof. Consider the $G$-action $g(x, h)=(x, g h)$ on $X \times G . \quad(X \times G$, $\widetilde{S})$ is a $G$-extension of $(X, S)$. Corollary 1 follows from Theorem 1 .

q.e.d.

\section{§ 2. Ergodicity of a G-extension}

Lemma 3. (W. Parry [6]). Let $(\Omega, \mu, \varphi)$ be a measure-preserving dynamics such that $\varphi(g \omega)=\sigma(g) \varphi(\omega)$ for $\omega$ in $\Omega$ and $g$ in $G$ for some automorphism $\sigma$ of $G$.

Let $V_{r}(\gamma \in \widehat{G})$ be the set of all $f_{\gamma}$ in $L^{2}(\Omega, \mu)$ such that $f_{r}(g \omega)$ $=r(g) f_{r}(\omega)$ with $\omega$ in $\Omega$ and $g$ in $G$. Then

(1) $L^{2}(\Omega, \mu)=\sum_{r \in \hat{G}} \oplus V_{r}$ (orthogonal sum) and

(2) if $f_{r}$ is in $V_{r}$ then $f_{r} \varphi$ is in $V_{r \sigma}$. 
Proof. (1) For an $f_{r}$ in $V_{r}$ and an $f_{\gamma^{\prime}}$ in $V_{r^{\prime}}$ we have

$$
\begin{aligned}
\int f_{r}(\omega) \overline{f_{r^{\prime}}(\omega)} d \mu(\omega) & =\int f_{r}(g \omega) \overline{f_{r^{\prime}}(g \omega)} d \mu(\omega) \\
& =\gamma(g) \overline{\gamma\left(g^{\prime}\right)} \int f_{r}(\omega) \overline{f_{r^{\prime}}(\omega)} d \mu(\omega) .
\end{aligned}
$$

If $r \neq \gamma^{\prime}, \gamma(g) \overline{\gamma^{\prime}(g)} \neq 1$ for some $g$ in $G$, and so $f_{\gamma}$ is orthogonal to $f_{\gamma^{\prime}}$. Suppose that $f$ in $L^{2}(\Omega, \mu)$ is orthogonal to any function in $\underset{r \in \hat{G}}{\cup} V_{r}$. Put $f_{r}(\omega)=\int \gamma(g) f\left(g^{-1} \omega\right) d g$ for $\gamma$ in $\widehat{G}$, then $f_{r}$ is in $V_{r}$. We have

$$
\begin{aligned}
\int f_{r}(\omega) \overline{f_{r}(\omega)} d \mu(\omega) & =\int f_{r}(\omega) \int \overline{r(g) f\left(g^{-1} \omega\right)} d g d \mu(\omega) \\
& =\iint f_{r}\left(g^{-1} \omega\right) \overline{f\left(g^{-1} \omega\right)} d \mu(\omega) d g \\
& =\int f_{r}(\omega) \overline{f(\omega)} f \mu(\omega)=0 .
\end{aligned}
$$

Hence $f_{r}(\omega)=0$ for $\omega$ in $\Omega$ and $\gamma$ in $\widehat{G}$, and thus $f(\omega)=0$ for $\omega$ in $\Omega$. Assersion (2) follows from the equation

$$
f_{r}(\varphi g \omega)=f_{r}(\sigma(g) \varphi \omega)=\gamma(\sigma(g)) f_{r}(\varphi \omega)
$$

q.e.d.

Theorem 2. Let $(\Omega, \mu, \varphi)$ be a G-extension of type $\sigma$ of an ergodic measure-preserving dynamics. Then $\varphi$ is not ergodic if and only if there exists a positive integer $n$ and $a r$ in $\widehat{G}, n$-periodic with respect to $\sigma$ and not equal to 1 , and an $f_{r}$ in $L^{2}(\Omega, \mu), f_{r} \neq 0$, such that $f_{r}\left(\varphi^{n}(\omega)=f_{r}(\omega)\right.$ and $f_{r}(g \omega)=\gamma(g) f_{r}(\omega)$, for $\omega$ in $\Omega$ and $g$ in $G$.

Proof. Let $f_{r}$ be a function which satisfies the conditions of Theorem 2. Put $f(\omega)=f_{r}(\omega)+f_{r}(\varphi \omega)+\cdots+f_{r}\left(\varphi^{n-1} \omega\right)$. Then $f$ is in $V_{r} \oplus V_{r \sigma} \oplus$ $\cdots \oplus V_{r \sigma^{n-1}}$ and $f(\varphi \omega)=f(\omega)$ for $\omega$ in $\Omega$. That is, $f$ is not constant and $\varphi$-invariant. Hence $\varphi$ is not ergodic. Conversely, let $f$ be a not constant function drawn from $L^{2}(\Omega, \mu)$ such that $f \varphi=f$, and let $f=\sum_{r \in \hat{G}} \oplus f_{r}$ with $f_{r}$ in $V_{r}$ be the direct sum decomposition of $f$. Then $f \varphi=\sum_{r \in \hat{G}} \oplus f_{r} \varphi$ where $f_{r} \varphi$ is in $V_{r \sigma}$. From $f \varphi=f$ we have $f_{\tau} \varphi=f_{r \sigma}$ and $\left\|f_{r}\right\|_{L^{2}}=\left\|f_{r \sigma}\right\|_{L^{2}}$ for $\gamma$ in $\widehat{G}$. From the orthogonality of $f_{r}$ 's we have $f_{r}=0$ if $\gamma$ is not periodic w.r.t. $\sigma$. Since any $\varphi$-invariant, $G$-invariant function is constant 
from the ergodic assumption, there exists a positive integer $n$ and an $n$-periodic $\gamma$ in $\widehat{G}, \gamma \neq 1$ such that $f_{r} \neq 0$. We have $f_{r}\left(\varphi^{n} \omega\right)=f_{r^{n}}(\omega)=f_{r}(\omega)$ for $\omega$ in $\Omega$.

q.e.d.

Corollary 2. Let $(\Omega, \mu, \varphi)$ be an ergodic measure-preserving dynamics, $\alpha(\omega)$ be a measurable G-valued function and $\sigma$ be an automorphism of $G$. $\widetilde{\varphi}$ is a measure-preserving transformation of the product $\Omega \times G$ defined by

$$
\widetilde{\varphi}(\omega, g)=(\varphi \omega, \alpha(\omega) \sigma(g)), \quad(\omega, g) \text { in } \Omega \times G .
$$

Then $(\Omega \times G, \mu \times d g, \widetilde{\varphi})$ is not ergodic if and only if there exists a positive integer $n$ and $a \gamma$ in $\widehat{G}, n$-periodic with respect to $\sigma$ and not equal to 1 , and an $f$ in $L^{2}(\Omega, \mu), f \neq 0$ such that $\gamma\left(\alpha\left(\varphi^{n-1} \omega\right) \sigma\left(\alpha\left(\varphi^{n-2} \omega\right)\right)\right.$ $\left.\cdots \sigma^{n-1}(\alpha(\omega))\right) f\left(\varphi^{n} \omega\right)=f(\omega)$ for $\omega$ in $\Omega$.

Proof. Consider the $G$-action $g(\omega, h)=(\omega, g h)$ on $\Omega \times G . \quad(\Omega \times G$, $\mu \times d g, \widetilde{\varphi})$ is a $G$-extension of type $\sigma$ of $(\Omega, \mu, \varphi)$. Corollary 2 follows from Theorem 2.

q.e.d.

Corollary 3. (1) When $\sigma$ of Corollary 2 is the identity, $\widetilde{\varphi}$ is not ergodic if and only if there exists $a \gamma$ in $\widehat{G}, \gamma \neq 1$, and a measurable function $f$ such that $|f(\omega)|=1$, and $\gamma(\alpha(\omega)) f(\varphi \omega)=f(\omega)$ for $\omega$ in $\Omega$. (H. Anzai [1]).

(2) When $\alpha(\omega)=h$ for $\omega$ in $\Omega$ and $G$ is connected, $\widetilde{\varphi}$ of Corollary 2 is not ergodic if and only if (i) there exists an $n \geqq 2$ and an nperiodic $\gamma$ in $G$, or (ii) there exists a 1-periodic $\gamma$ in $\widehat{G}, \gamma \neq 1$, and a measurable function $f$, such that $|f(\omega)|=1$ and $\gamma(h) f(\varphi \omega)=f(\omega)$ for $\omega$ in $\Omega$, that is, $\gamma(h)^{-1}$ is in the point spectrum of $\varphi$.

Proof. (1) Clear from Corollary 2.

(2) If $n \geqq 2$ and $\gamma$ be $n$-periodic, put $\gamma_{1}=\frac{\gamma \sigma}{\gamma}$. Then $\gamma_{1}$ is in $\widehat{G}, \gamma_{1} \neq 1$ and $\gamma_{1} \sigma^{n}=\gamma_{1}$. Let $n_{1}$ be the period of $\gamma_{1}$; we may represent $n$ as $n_{1} p$ where $p$ is a positive integer. If $\frac{\gamma_{1}^{p} \sigma^{k}}{\gamma_{1}{ }^{p}}=\left(\frac{\gamma_{1} \sigma^{k}}{\gamma_{1}}\right)^{p}=1$ for a positive integer $k$, we have $\frac{\gamma_{1} \sigma^{k}}{\gamma_{1}}=1$ from the connectedness of $G$. This means that $\gamma_{1}{ }^{p}$ 
is also $n_{1}$-periodic. Since $\gamma_{1}^{p}\left(h \sigma h \cdots \sigma^{n_{1}-1} h\right)=\gamma_{1}\left(h \sigma h \cdots \sigma^{n-1} h\right)=1, \gamma_{1}^{p}(h \sigma h \cdots$ $\left.\sigma^{n_{1}-1} / 2\right) f\left(\varphi^{n_{1}-1} \omega\right)=f(\omega)$ for any constant function $f$. The rest of the proof is obvious.

q.e.d.

Corollary 4. (1) The affine transformation $g \mapsto h \sigma(g)$ on connected $G$ is not ergodic if and only if there exists an n-periodic $\gamma$ in $\widehat{G}$ with $n \geqq 2$ or there exists a 1-periodic $\gamma$ in $\widehat{G}, \gamma \neq 1$ with $\gamma(h)=1$ (F. Hahn [3]).

(2) The group automorphism $g \mapsto \sigma(g)$ on $G$ is not ergodic iff there exists an n-periodic $\gamma$ in $\widehat{G}, r \neq 1$ for some $n \geqq 1$. (P.R. Halmos [4]). (3) The translation $g \mapsto h g$ on $G$ is not ergodic iff there exists a $\gamma$ in $\widehat{G}, \gamma \neq 1$ with $\gamma(h)=1$.

\section{Acknowledgement}

A part of this work has been made during the author's visit to the University of Heidelberg. The author would like to thank the members of the department of Mathematics for their warm hospitality.

\section{References}

[1] Anzai, H., Ergodic skew product transformation on the torus, Osaka Math. J., 3 (1951), 83-99.

[2] Fürstenberg, H., Strict ergodicity and transformations of the torus. Amer, J. Math., 83 (1961), 573-601.

[3] Hahn, F., On affine transformations of compact abelian groups, Amer. J. Math., 85 (1963), 428-446.

[4] Halmos, P. R., On automorphisms of compact groups, Bull. Amer. Math. Soc., 49 (1943), 619-624.

[5] Parry, W., Compact abelian group extensions of discrete dynamical systems, $Z$. Wahrscheinlichkeitstheorie und Verw. Gebiete, 13 (1969), 95-113.

[6] Parry, W., Spectral analysis of $G$-extensions of dynamical systems, Topology, 9 (1970), 217-224. 
